\title{
Diseño del área quirúrgica para experimentación animal; cinco casos de estudio
}

\section{Design of the surgical area for animal research; five case studies}

\author{
$\underline{\text { Aitziber Goikoetxea Ibarrondo }}^{(*)}$, Pedro Berroya Ortiz de Urbina ${ }^{(* *)}$, Mercedes Valiente López ${ }^{(* * *)}$, Francisco Domouso de Alba ${ }^{(* * * *)}$
}

\section{RESUMEN}

Las unidades de cirugía para experimentación animal son instalaciones complejas de planificar y diseñar. Es por ello que para afrontar un proyecto de este tipo resulta recomendable visitar o recopilar la información que se encuentra disponible sobre unidades similares ya construidas, para así poder analizar los diferentes modelos conceptuales y los flujos de trabajo que puedan servir de referencia. En este trabajo, a través de expresiones gráficas, se estudia el modelo organizativo, diseño conceptual y características constructivas de las unidades quirúrgicas experimentales de cinco Institutos de Investigación Sanitaria Acreditados por el Instituto de Salud Carlos III. A partir de la puesta en común de los resultados obtenidos del análisis de varios centros se pretende establecer unas pautas de comportamiento o protocolos que puedan ser de utilidad a los profesionales responsables del diseño, construcción y puesta en funcionamiento de nuevos quirófanos experimentales.

Palabras clave: experimentación animal grande; cirugía experimental; diseño de quirófanos experimentales; prácticas quirúrgicas; investigación biomédica.

\section{ABSTRACT}

The surgical units for animal research are complex facilities for planning and designing. That is why in order to deal with a project of this type it is advisable to visit or gather the information that is available about similar units already built in order to analyze the different conceptual models and workflows that could serve as a reference. In this work, we studied the schematic floor plans, the organizational model, conceptual design and construction features of the experimental surgical unit of five Institutes of Health Research accredited by the Carlos III Health Institute are studied. Based on the sharing of the results obtained from the analysis of several units, it is intended to establish behavioral guidelines or protocols that may be useful to the professionals responsible for the design, construction and operation of new experimental operating rooms.

Keywords: large animal research; experimental surgery; experimental operating rooms design; surgical practices; biomedical research.

(*) Ingeniera de Edificación. Universidad Politécnica de Madrid (España).

${ }^{* *}$ Ingeniero de Edificación. Universidad Politécnica de Madrid (España).

(***) Dra. Arquitecta. Catedrática de Dibujo Arquitectónico. Universidad Politécnica de Madrid, Madrid (España).

(****) Dr. Arquitecto. Universidad Europea de Madrid, Madrid (España).

Persona de contacto/Corresponding author: aitziber.goikoetxea@gmail.com (A. Goikoetxea)

ORCID: https://orcid.org/oooo-0001-5884-9187 (A. Goikoetxea); https://orcid.org/oooo-0001-9957-903X (P. Berroya);

https://orcid.org/oooo-0oo1-8643-7921 (M. Valiente); https://orcid.org/oooo-0oo2-0392-9582 (F. Domouso)

Cómo citar este artículo/Citation: Goikoetxea Ibarrondo, Aitziber; Berroya Ortiz de Urbina, Pedro; Valiente López, Mercedes; Domouso de Alba, Francisco (2020). Diseño del área quirúrgica para experimentación animal; cinco casos de estudio. Informes de la Construcción, 72 (560): e366. https://doi.org/10.3989/ic.75050.

Copyright: (c) 2020 CSIC. Este es un artículo de acceso abierto distribuido bajo los términos de la licencia de uso y distribución Creative Commons Reconocimiento 4.0 Internacional (CC BY 4.0). 


\section{INTRODUCCIÓN}

Los Institutos de Investigación Sanitaria acreditados son el resultado de la asociación a los hospitales docentes e investigadores del Sistema Nacional de Salud, de Universidades y otros centros públicos y privados de investigación. Son instituciones con entidad jurídica propia, independiente al Hospital, pero cuyas instalaciones habitualmente están dentro del ámbito hospitalario, siendo los investigadores trabajadores del propio hospital.

Su objetivo principal es realizar investigación traslacional, de manera que todo el conocimiento generado a partir de la investigación biomédica de excelencia se traduzca, finalmente, en el tratamiento y prevención de enfermedades, en definitiva, en la mejora de la salud y calidad de vida de la población.

Entre las diferentes plataformas que componen estos institutos se encuentra el Centro de Experimentación Animal, que es toda instalación, edificio, grupo de edificios u otros locales, incluidos aquellos no totalmente cerrados o cubiertos, así como las instalaciones móviles y todo el conjunto de medios personales y materiales organizados por su titular para la cría, el suministro o la utilización de animales de experimentación (1). Normalmente está compuesto por dos áreas específicas determinadas por el tamaño del animal con el que se va a experimentar: área de experimentación de animal pequeño y área de experimentación de animal grande.

Dentro del área de experimentación de animal grande se encuentra la unidad de cirugía experimental que desde el ámbito docente tiene como objetivo organizar y dar soporte a la realización de cursos de formación en técnicas quirúrgicas, microquirúrgicas, laparoscópicas, anestésicas, entre otros, utilizando para ello diferentes modelos animales.

El presente trabajo se centra en realizar un análisis pormenorizado del modelo organizativo y funcional, del diseño conceptual y de las características constructivas del área quirúrgica para experimentación animal de diferentes institutos de investigación sanitaria acreditados. Teniendo presentes los manuales y guías existentes para el planteamiento de este tipo de instalaciones, se analizan las características generales del área, los locales que lo componen, la organización y distribución planteada para la configuración de la unidad, los flujos principales de trabajo implantados, así como las soluciones constructivas adoptadas.

\section{OBJETIVOS}

Los quirófanos experimentales son salas de ambiente controlado complejas de planificar y diseñar. En general, a la hora de afrontar el proyecto de una nueva unidad, resulta recomendable visitar o recopilar la información que se encuentra disponible sobre unidades similares ya construidas, con el fin de analizar los diferentes conceptos de organización y flujos de trabajo establecidos que puedan servir de aplicación en la elaboración de esos nuevos proyectos.

Una instalación bien planeada, diseñada y construida, así como adecuadamente mantenida y gestionada es fundamental para el cuidado y uso de animales de experimentación, ya que facilita el funcionamiento eficiente, económico y seguro (2). Es por ello que se considera interesante el presente trabajo, ya que el objetivo principal es que los resultados obte- nidos del análisis de diferentes casos puedan servir de guía o referencia; en primer lugar, a los profesionales responsables de los centros de experimentación animal, por ser habitualmente los encargados de elaborar el plan funcional de partida; en segundo lugar, a los profesionales de la arquitectura e ingeniería, como responsables de la redacción y ejecución de los proyectos de construcción de las infraestructuras; y en tercer lugar, a los investigadores, por ser los usuarios finales de las instalaciones diseñadas.

\section{METODOLOGÍA}

Con la intención de investigar los centros de vanguardia en el ámbito de la investigación biomédica, el presente trabajo se ha centrado en el estudio de las unidades quirúrgicas experimentales de los Institutos de Investigación Sanitaria Acreditados por el Instituto de Salud Carlos III (3).

Entre los 31 institutos acreditados a fecha julio 2019 (4), para este estudio se han seleccionado las siguientes cinco unidades quirúrgicas visitadas y pertenecientes a Institutos de Investigación Sanitaria Acreditados: Instituto de Investigación Hospital 12 de Octubre (i+12, Madrid), Instituto Ramón y Cajal de Investigación Sanitaria (IRYCIS, Madrid), Instituto de Investigación Sanitaria Biocruces (IIS Biocruces, Bizkaia), Instituto de Investigación Sanitaria Biodonostia (IIS Biodonostia, Gipuzkoa), Instituto de Investigación Sanitaria Aragón (IISA, Aragón).

A partir de la documentación obtenida de las visitas realizadas a los institutos, se realizó un análisis detallado de las características generales, de la organización y diseño, así como de las características constructivas del área de cirugía experimental. Para facilitar la puesta en común de los datos obtenidos, se han elaborado unas tablas en las que quedan identificados, por una parte, las características comunes a los institutos, y por la otra, las características específicas de ellos.

Por otra parte, a partir de la documentación gráfica de la distribución de plantas se elaboraron unos planos estandarizados que reflejan la organización y distribución de las diferentes áreas, la identificación de los locales que lo conforman, así como los flujos de trabajo establecidos para el suministro de materiales, animales, personal responsable del cuidado de los animales y personal investigador, así como la retirada de residuos.

Fruto de la investigación realizada, y tomando como base las tablas y representaciones gráficas estandarizadas, en este trabajo se analizan el modelo organizativo y funcional, el diseño conceptual y de las características constructivas de cinco unidades de cirugía experimental de institutos de investigación sanitaria acreditados.

\section{RESULTADOS}

La actividad mayoritaria en el área de experimentación de animal grande es la cirugía experimental, actividad que nos encontramos en todos los institutos de investigación sanitaria. Es por ello que el presente trabajo se ha centrado en contextualizar la complejidad del área de experimentación de animal grande y específicamente en realizar un análisis pormenorizado del área quirúrgica experimental.

Este servicio habitualmente está diseñado para prestar apoyo en los procedimientos quirúrgicos necesarios para la investi- 
gación y proveer tanto del material como de las técnicas quirúrgicas para la formación de los profesionales del sistema sanitario en cualquiera de las especialidades quirúrgicas y medico quirúrgicas.

Para la realización de esas prácticas quirúrgicas resulta necesario por una parte disponer de modelos animales que se asemejen al ser humano, para lo cual resulta necesario disponer de un animalario donde poder estabular las especies con las que se va a experimentar (5); y, por la otra, disponer de unos quirófanos en el que los profesionales puedan realizar las prácticas quirúrgicas en condiciones lo más similares posibles a las que se van a encontrar a posteriori en el ámbito hospitalario. En general, los animales estabulados en el centro deberán ser trasladados al área quirúrgica, para una vez finalizada la intervención volver a la zona de estabulación animal o en el caso de que sean terminales, a la zona de almacenaje de residuos animales.

En el diseño del conjunto de instalaciones para experimentación animal, con el objetivo de garantizar el bienestar animal, es fundamental tener presente la normativa de aplicación para la protección de los animales utilizados con fines de experimentación, incluyendo la educación y docencia (6).

\subsection{Locales que componen la unidad quirúrgica experimental}

El programa funcional y la especificación de los recursos necesarios para el desarrollo de la actividad del área quirúrgica dependen especialmente de las decisiones que los responsables de la gestión del instituto hayan adoptado en relación a las líneas de investigación y necesidades del centro, a la definición de procesos y protocolos de funcionamiento, así como a las prioridades de la investigación establecidas; que tendrán que adaptarse a cada realidad concreta, así como a los condicionantes específicos de cada situación. En cuanto a los locales necesarios para el desarrollo de la actividad, la mayoría de los programas quirúrgicos contemplan las siguientes salas:

Vestuarios: los vestuarios de cambio para el personal que va a acceder al área quirúrgica, separados por sexo para uso del personal que acceda o trabaje en el área quirúrgica, con una zona de aseo y taquillas para facilitar a los usuarios el cambio de ropa de calle por otra de uso específico para esta zona.

Preparación médica: el área de preparación médica para desinfección y colocación de mascarilla del personal previo acceso a los quirófanos, siendo recomendable ubicarlo contiguo al quirófano para tener una visión del quirófano que permita el control de la actividad que se desarrolla en él, a través de las ventanas de visualización. El equipamiento de esta sala es el necesario para realizar el lavado de manos con grifo quirúrgico de accionamiento no manual, dispensadores antisépticos, secador de manos automático y reloj. Además, es habitual disponer de espacio para la colocación de estantes para guardar el material desechable de protección como son los guantes, gorros, máscaras, etc., así como la recogida de envoltorios de papel.

Zona de preparación animal: como zona destinada a preparar el animal previo acceso al quirófano en el que se realizan tareas tales como el rasurado, limpieza, desinfección, incluso anestesiado, para una vez preparado el animal trasladarlo directamente al quirófano.

Quirófanos: el local donde sea realiza la intervención quirúrgica y dotado del equipamiento necesario para el desarrollo de la actividad tales como las torres de cirugía y anestesia, lámparas quirúrgicas, mesas de operaciones, paneles técnicos y equipos de retransmisión de imágenes, entre otros.

Sala de recuperación postoperatoria: local donde ingresan los animales no terminales que han sido sometidos a una intervención quirúrgica con anestesia general, regional o sedación, y en el que recibirán los cuidados necesarios hasta su traslado al área de estabulación.

Procedimientos: se concibe como una zona de apoyo al área quirúrgica para la realización de procedimientos experimentales, necropsias, tratamiento clínico o procedimientos de laboratorio de diagnóstico, entre otros.

Sala de esterilización y lavado: para el acondicionamiento, limpieza y esterilización del material empleado en los quirófanos experimentales, con los equipos e instalaciones necesarias para el funcionamiento, así como estantes para el almacenamiento del material esterilizado.

Almacén de equipos limpio: destinados al almacenamiento de material limpio que se emplee en los quirófanos, material de farmacia y fungible, así como para la ubicación de aquellos equipos portátiles que no necesiten estar de manera permanente en el interior de los quirófanos tales como los de radiodiagnóstico e intensificadores de imagen, láser quirúrgico, respirador y lámpara portátil. La programación de estos locales posibilita un mantenimiento y limpieza adecuado de los quirófanos, además de disponer de circulaciones libre de obstáculos. Su número, disposición y tamaño se encuentra relacionado con la actividad quirúrgica programada, dimensión y diseño del bloque y por el tipo de intervenciones por especialidades.

\subsection{Criterios de organización de la unidad quirúrgica experimental}

El quirófano experimental es el local en el que se realiza la intervención quirúrgica y donde confluyen los distintos tráficos de la unidad: los animales, el personal, el material estéril, el material usado y los residuos. La importancia de la organización y distribución de los quirófanos, así como de los locales de servicio principales que componen el área radica en que definirá las diferentes circulaciones asociadas, y por lo tanto el grado de asepsia de la unidad.

En estas áreas quirúrgicas para experimentación animal, el uso de los quirófanos es una actividad programada en la que la circulación de los animales está protocolizada. El modelo animal más utilizado es el cerdo o cerdo en miniatura (7), siendo lo habitual que los animales sean terminales, o bien que interese ver la evolución durante unas horas determinadas sin tener que estabularlos durante un periodo largo de tiempo.

A pesar de que no existe una normativa específica para los quirófanos experimentales que determine el esquema u organización interna para el desarrollo de estas unidades quirúrgicas, sí podemos encontrar, por una parte, guías o manuales para el diseño 
de los quirófanos asistencial $(8,9), \mathrm{y}$, por la otra, manuales para la organización del área de cirugía experimental $(2,10,11,12)$.

Entre las diferentes guías existentes para analizar la organización del área quirúrgica experimental, hemos tomado como referencia los criterios de distribución en planta y los flujos de trabajo establecidos en una de las guías publicadas (Figura 1). En el esquema observamos cómo toda la organización se desarrolla a partir de la implantación de la sala de operaciones, de manera que colindante a ella se distribuyen los diferentes locales para acceso de personal, animales y materiales. Esta disposición de los locales hace la función de esclusa y permite evitar la contaminación de agentes patógenos entre áreas, ya que favorece el control de las diferencias de presión entre zonas y posibilita asegurar que el flujo de aire circule en la dirección adecuada. Atendiendo a los flujos de trabajo observamos tres circuitos diferentes: uno para los animales que desde al área de estabulación animal son trasladados al quirófano previo paso por una zona de preparación animal, para una vez finalizada la intervención quirúrgica, volver a la zona de recuperación postanestésica, o, si el animal es terminal, trasladarlo al área de almacenamiento de residuo animal; un segundo circuito para el personal médico que accede y sale del quirófano previo paso por la zona de preparación médica para la colocación del atuendo y realizar el lavado de manos; y, un tercero para introducir el material quirúrgico esterilizado a los quirófanos.

Por otra parte, tomando como referencia las recomendaciones de organización de las áreas quirúrgicas hospitalarias observamos que existe un consenso de criterios de separación del área quirúrgica por zonas en función del mayor o menor riesgo de infección. De esta manera, tomando como referencia el grado de asepsia necesaria en cada zona que componen el área quirúrgica hospitalaria y asimilándolo al área quirúrgica experimental, nos encontramos que con el fin de eliminar lo máximo posible las fuentes de contaminación y poder garantizar las condiciones ambientales necesarias en la mesa de operaciones, el área quirúrgica se divide en tres zonas principales de restricción progresiva:

- Zona Negra: es la primera zona de restricción que funciona como zona amortiguadora de protección, que comprende las zonas periféricas de administración, vestuarios y aseos. A esta zona se permite el acceso con bata clínica y es donde el personal se coloca el atuendo quirúrgico.
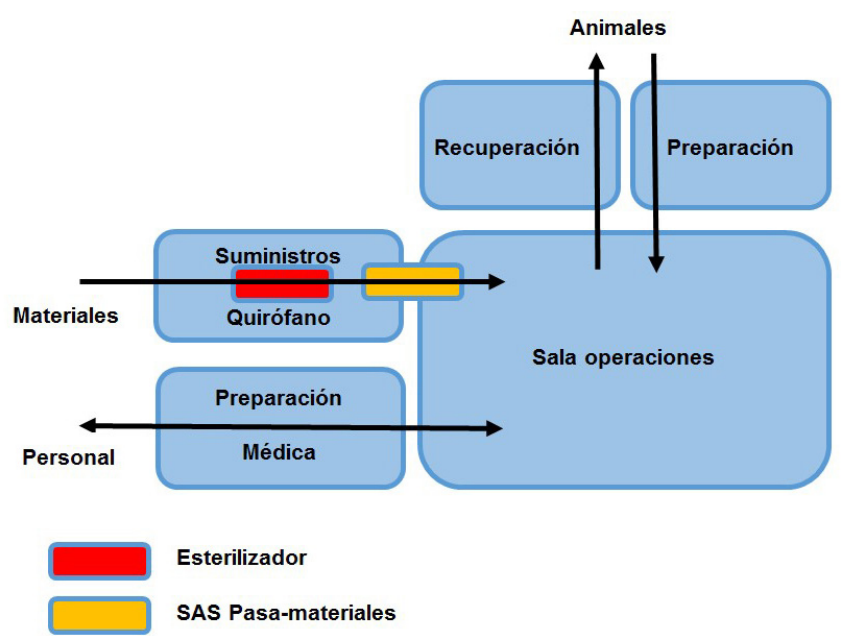

Figura 1. Flujos de trabajo quirófanos experimentales (10).
- Zona Gris: para acceder a esta zona se requiere portar el uniforme completo de pijama y comprende la sala de preparación médica, sala de preparación animal, sala de recuperación postanestésica, sala de equipos, y local para material estéril.

- Zona Blanca: es el área que comprende la sala de operaciones, la zona de mayor exigencia aséptica y por lo tanto la de mayor restricción.

Uno de los temas en los que no existe una clara determinación, y sobre el que existe una disparidad de opiniones es en el criterio de diseño para el tránsito dentro de la unidad quirúrgica, de manera que podemos encontrar soluciones válidas bien realizadas mediante pasillo único o bien realizadas mediante pasillo doble. En este sentido, aunque el diseño de los bloques quirúrgicos hospitalarios ha estado basado en la necesidad de disponer de una circulación limpia y otra sucia, desde hace tiempo se ha demostrado que este criterio de segregación de pasillos es ajena a toda evidencia científica (8).

En el caso del pasillo único, se producen cruces del material limpio con el sucio y de los pacientes con los médicos. Este criterio de diseño conlleva la aplicación de un exhaustivo procedimiento de trabajo, entre los que se incluiría el uso de recipientes y contenedores seguros precintados. En cambio, en el caso de distribución mediante pasillo doble, el principio general es establecer una única dirección de flujo de manera que siempre se circule desde la zona limpia a la sucia, para que una vez esterilizados los materiales, puedan volver a la zona limpia. Tomando como base este principio existen diferentes posibilidades de organización de flujos de trabajo que quedan reflejados en los siguientes esquemas (Figura 2).

La implantación de diseño de un tipo u otro afecta directamente a la organización del personal y a la disciplina de trabajo, por lo que en su decisión estaría implicado tanto el equipo de diseño como los responsables del área de experimentación. Entre otros motivos, porque si bien desde el punto de vista de aprovechamiento de la edificabilidad y optimización de recursos, la solución de pasillo único es la más adecuada, esta opción requiere por parte de los profesionales del cumplimiento estricto de los procedimientos de trabajo establecidos.

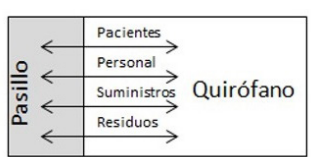

Pasillo único: los pacientes, el personal, los suministros y los residuos hacen uso del mismo pasillo, no existe segregación de circulaciones

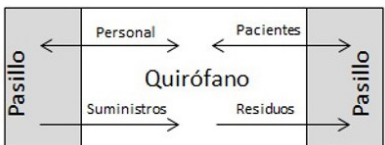

Doble Pasillo: los pacientes y los residuos son segregados del personal y los suministros

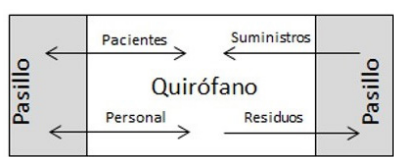

Doble Pasillo: el personal y los pacientes son segregados de los suministros y residuos
Figura 2. Política de segregación de circulaciones en quirófanos (13). 


\subsection{Representaciones gráficas de la configuración de las unidades quirúrgicas experimentales}

Se ha considerado que la mejor manera de realizar un análisis de la organización y distribución planteada es a través de la representación gráfica estandarizada de las áreas quirúrgicas que disponen los centros de experimentación animal de cada uno de los institutos investigados (Figuras 3-8).

Para ello, se ha identificado por código de colores el área funcional al que pertenece y por código numérico además de alfabetico el tipo de local que es. En los casos en los que los locales relacionados con el área quirúrgica experimental se ubican lejos de los quirófanos se ha optado por indicar mediante una flecha la dirección de su ubicación. Por otra parte, para localizar fácilmente los quirófanos, se ha optado por representar las mesas de operaciones. Con el fin de realizar una lectura sencilla de las circulaciones también se representan mediante lineas de diferentes colores y trazos los flujos de trabajo del personal médico, de los animales, así como del material limpio y de los residuos generados en el área quirúrgica. Junto a cada una de las representaciones gráficas, se hace una breve descripción de la configuración del área, de la disposición de los locales, de la tipología de distribución que se ha aplicado, así como del criterio de circulaciones establecidas.

El área quirúrgica del Instituto de Investigación Sanitaria Hospital 12 Octubre i+12 (Figura 4) se compone de un único quirófano doble, disponiendo de los locales de servicio alrededor del quirófano. Los vestuarios de personal son compartidos con el área de experimentación de animal pequeño y tienen acceso directo a la zona de preparación médica. Por otra parte, tanto la zona de preparación médica como el almacén de material estéril, que tienen acceso directo al quirófano, también son locales compartidos con el área de microcirugía destinado a la experimentación con animal pequeño. Esta configuración la consideramos como pasillo doble porque hay una separación de circulaciones; de manera que por una parte hay un punto común de acceso al quirófano para el personal médico, material estéril y animales; y otro punto de salida diferente para la retirada de residuos, si bien al final ambos recorridos confluyen en el mismo pasillo.

El área quirúrgica del Instituto Ramón y Cajal de Investigación IRYCIS (Figura 5) se compone de un núcleo compacto compuesto por dos quirófanos simples en el que se compar-

\begin{tabular}{l} 
ÁREAS FUNCIONALES \\
Experimentación animal grande \\
Experimentación animal pequeño \\
Áreas comunes a. pequeño y grande \\
Pasillos comunes \\
Elementos circulación vertical \\
Servicios generales \\
CIRCULACIONES \\
\hline Personal investigador \\
\hline Animales \\
\hline Suministro materiales
\end{tabular}

LOCALES UNIDAD QUIRÚRGICA

$\begin{array}{ll}\text { 1Q } & \text { Quirófano } \\ \text { 2PM } & \text { Preparación médica } \\ \text { 3PA } & \text { Preparación animal } \\ \text { 4AME } & \text { Almacén material estéril } \\ \text { 5V } & \text { Vestuarios } \\ \text { 6SLE } & 6 \text { Sala lavado y esterilización } \\ \text { 7A } & \text { Almacenes vários } \\ \text { 8P } & \text { Procedimientos } \\ \text { 9EAG } & \text { Estabulación animal grande }\end{array}$

Figura 3. Leyenda identificación de colores, numeración y líneas.

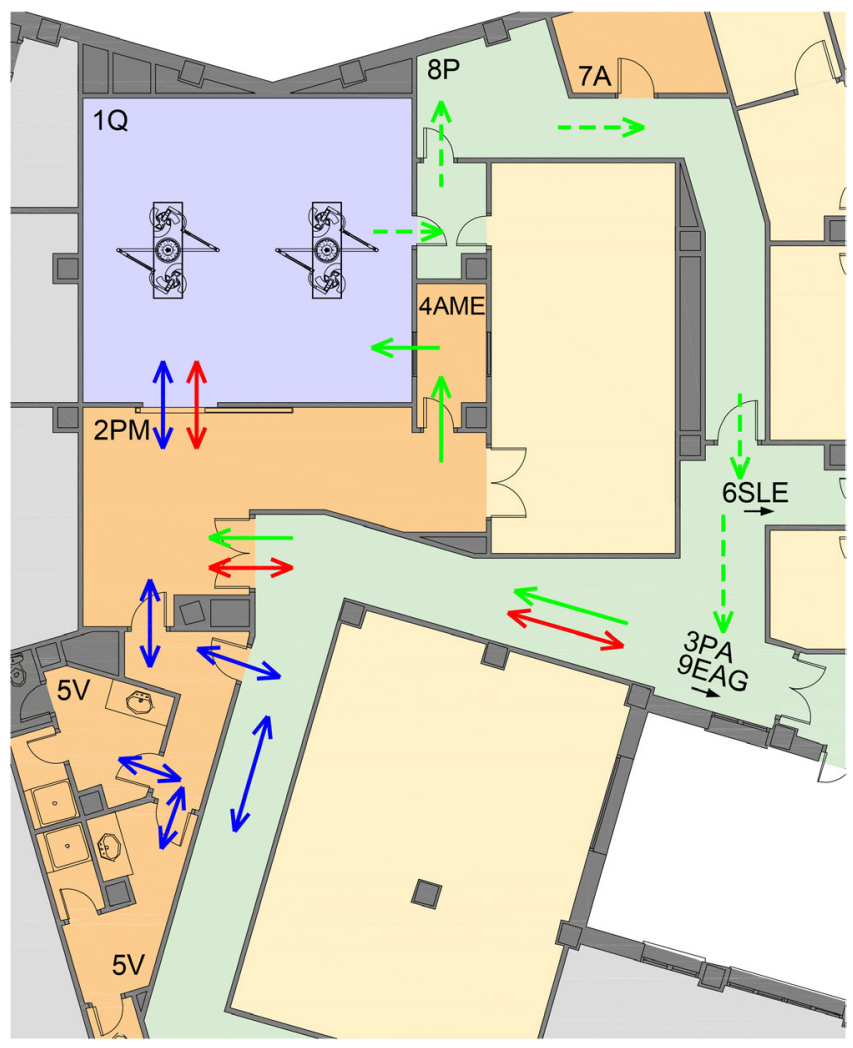

Figura 4. Organización área quirúrgica i+12.

ten para ambos quirófanos los locales de servicio tales como el almacén de material estéril, zona de preparación médica y preparación animal. El vestuario, el área de lavado y esterilización, así como el almacenamiento de residuos son locales de servicio compartidos con el animal pequeño. En este caso se dispone de un único pasillo de distribución, de manera que para poder acceder o salir del quirófano, tanto el personal médico, como los animales como los materiales están obligados a pasar por la zona de preparación médica.

El área quirúrgica del Instituto de Investigación Sanitaria Biodonostia IISBiodonostia (Figura 6) se compone de un quirófano con dos mesas. En este caso la zona de preparación

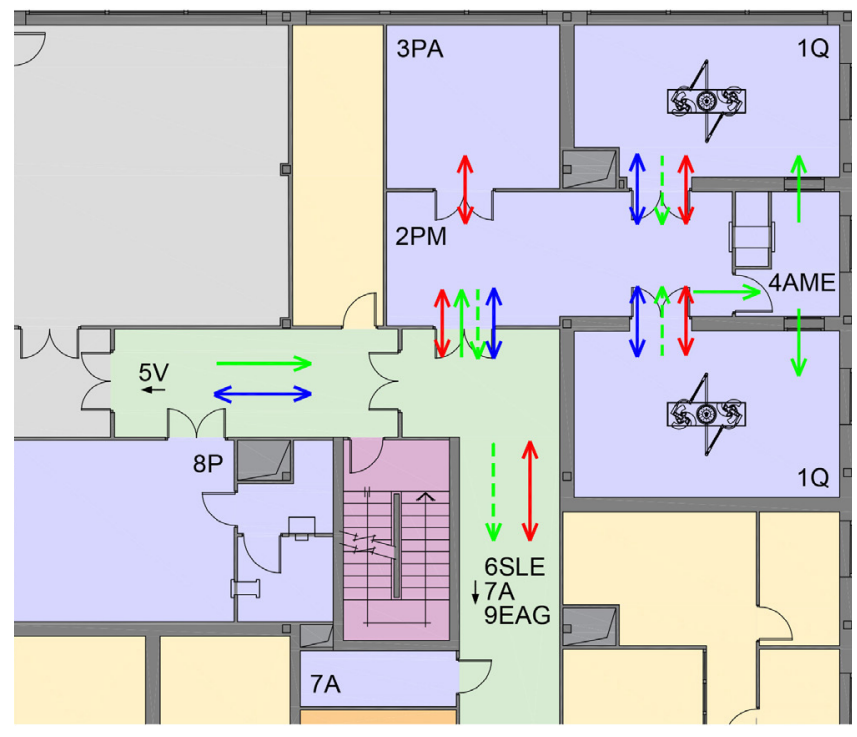

Figura 5. Organización área quirúrgica IRICYS. 


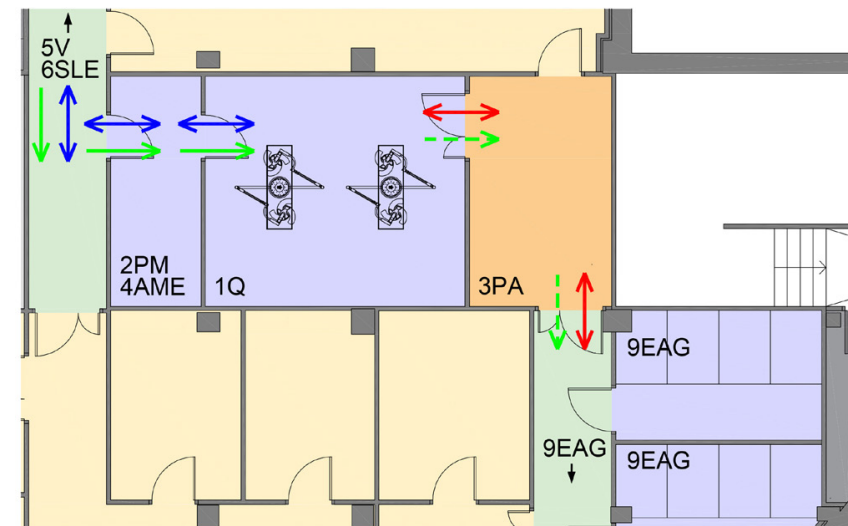

Figura 6. Organización área quirúrgica IISBiodonostia.

médica es compartida con el almacén de material estéril, y la zona de preparación animal es compartida tanto para el animal grande como para el pequeño. Los vestuarios de personal no son de uso exclusivo del área quirúrgica y es necesario discurrir por pasillos de uso común al animal grande y pequeño para llegar a la zona de preparación médica. El área de lavado y esterilización, así como el almacenamiento de residuos son locales de servicio también compartidos con el animal pequeño. En este caso se disponen de dos pasillos de distribución; uno para el personal médico y material limpio; y, otro para los animales y la retirada de los residuos.

El área quirúrgica del Instituto de Investigación Sanitaria Aragón IISA (Figura 7) se compone de tres quirófanos: dos simples y uno doble. Los quirófanos dobles comparten la zona de preparación médica con acceso directo a cada quirófano. Además, dispone de vestuarios de personal propios con acceso directo a la unidad, de un local destinado a almacenamiento de equipos quirúrgicos dentro del área, y una sala de lavado y esterilización, también de uso exclusivo del área quirúrgica. En este caso la distribución planteada la hemos considerado de pasillo doble porque hay una segregación de circulaciones para acceder al quirófano que garantiza unas condiciones asépticas concretas. De esta manera, por una parte, acceden los animales y salen los residuos; y, por la otra, acceden el personal médico y el material estéril, si bien es cierto que al final todas las circulaciones se mezclan en el pasillo exterior de la unidad quirúrgica.

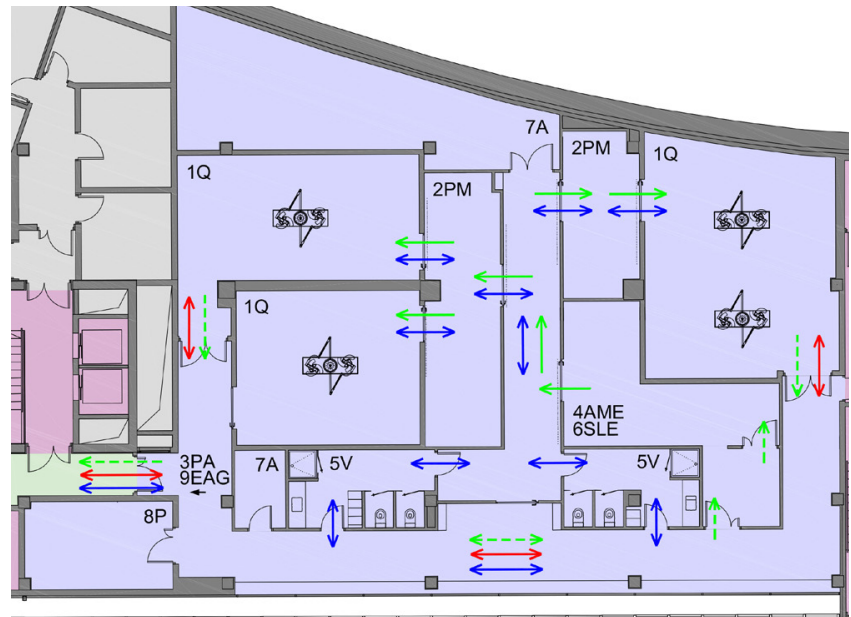

Figura 7. Organización área quirúrgica IISA.

El área quirúrgica del Instituto de Investigación Sanitaria Biocruces IISBiocruces (Figura 8) se compone de dos quirófanos, uno simple y uno con dos mesas de operaciones. Ambos quirófanos comparten la zona de preparación médica desde la que se accede a ambos quirófanos y en la que también se almacena el material estéril. La zona de preparación animal en cambio, se ubica colindante al quirófano simple, de manera que para acceder al quirófano doble es necesario pasar por el quirófano simple. Existen vestuarios de personal propios para acceso directo al área quirúrgica, el área de esterilización y lavado también es de uso exclusivo del área quirúrgica y se dispone de un local propio destinado al almacenamiento de equipos quirúrgicos. En este caso se dispone de dos pasillos diferenciados de distribución; uno para el personal médico y material limpio; y, otro para los animales y la retirada de los residuos.

\subsection{Análisis de la organización de la unidad quirúrgica experimental}

Con el fin de realizar una lectura sencilla de la organización de las áreas quirúrgicas experimentales de los institutos de investigación sanitaria, se ha elaborado una tabla en la que se hace una puesta en común de los resultados obtenidos (Tabla 1). En ella se listan los parámetros que definen el criterio organizativo

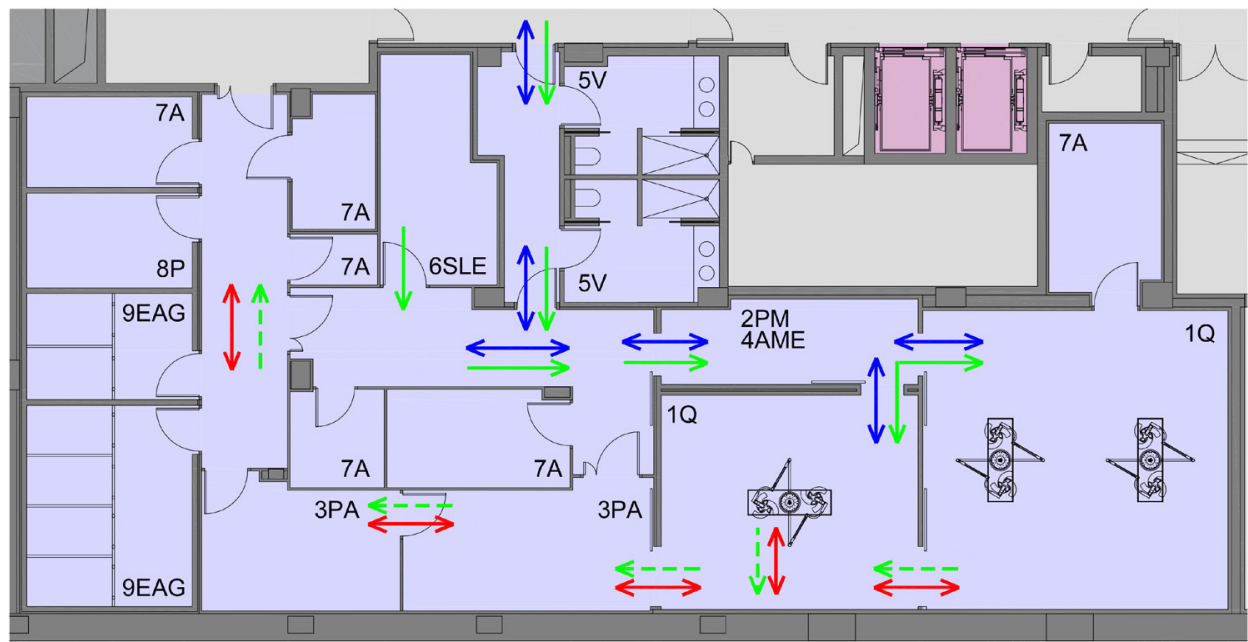

Figura 8. Organización área quirúrgica IISBiocruces. 
Tabla 1. Resumen toma datos organización y distribución del área quirúrgica experimental.

\begin{tabular}{|c|c|c|c|c|}
\hline Parámetros & $\begin{array}{l}\text { Características } \\
\text { comunes }\end{array}$ & $\begin{array}{c}\text { Institutos con } \\
\text { características } \\
\text { comunes }\end{array}$ & $\begin{array}{l}\text { Características } \\
\text { específicas }\end{array}$ & $\begin{array}{c}\text { Institutos con } \\
\text { características } \\
\text { específicas } \\
\end{array}$ \\
\hline $\begin{array}{l}\text { Tipo de planta en la } \\
\text { que se ubica }\end{array}$ & A cota cero; cota de acceso & $\begin{array}{l}\text { i+12, IRYCIS, } \\
\text { IIBiodonostia, } \\
\text { IISBiocruces, IISA }\end{array}$ & & \\
\hline $\begin{array}{l}\text { Independizado del } \\
\text { área quirúrgica } \\
\text { animal pequeño }\end{array}$ & No & $\begin{array}{l}\text { i+12, IRYCIS, } \\
\text { IISBiodonostia }\end{array}$ & $\mathrm{Si}$ & IISBiocruces, IISA \\
\hline $\begin{array}{l}\text { Zonas compartidas } \\
\text { con animal pequeño }\end{array}$ & $\begin{array}{l}\text { Pasillos, vestuarios, } \\
\text { almacenes varios y sala } \\
\text { lavado y esterilización }\end{array}$ & $\begin{array}{l}\text { IISBiodonstia, IRYCIS, } \\
i+12\end{array}$ & $\begin{array}{l}\text { Preparación animal } \\
\text { Preparación médica } \\
\text { No se comparten áreas }\end{array}$ & $\begin{array}{l}\text { IISBiodonostia } \\
\mathrm{i}+12 \\
\text { IISBiocruces, IISA }\end{array}$ \\
\hline $\mathrm{N}^{\circ}$ quirófanos & $2 \mathrm{Ud}$ & IRYCIS, IISBiocruces & $\begin{array}{l}1 \mathrm{Ud} \\
>2 \mathrm{Ud} \\
\end{array}$ & $\begin{array}{l}\text { IISBiodonostia, } \mathrm{i}+12 \\
\text { IISA }\end{array}$ \\
\hline Tipo de quirófanos & Doble & $\begin{array}{l}\text { i+12, } \\
\text { IISBiodonostia, } \\
\text { IISBiocruces, IISA }\end{array}$ & Simple & IRYCIS, IISBiocruces \\
\hline $\begin{array}{l}\text { Ubicación vestuarios } \\
\text { en área quirúrgica }\end{array}$ & $\begin{array}{l}\text { Contiguos al área } \\
\text { quirúrgica }\end{array}$ & i+12, IISBiocruces, IISA & En zonas comunes & $\begin{array}{l}\text { IRYCIS, } \\
\text { IISBiodonostia }\end{array}$ \\
\hline $\begin{array}{l}\text { Área preparación } \\
\text { médica }\end{array}$ & Exclusiva para personal & $\begin{array}{l}\text { IISBiodonostia, } \\
\text { IISBiocruces, IISA }\end{array}$ & $\begin{array}{l}\text { Compartida con acceso } \\
\text { animales }\end{array}$ & $\mathrm{i}+12$, IRYCIS \\
\hline Local material estéril & $\begin{array}{l}\text { Compartido con zona } \\
\text { preparación médica }\end{array}$ & $\begin{array}{l}\text { IISBiodonostia, } \\
\text { IISBiocruces, IISA }\end{array}$ & $\begin{array}{l}\text { Transfer materiales con } \\
\text { acceso directo a quirófanos }\end{array}$ & $\mathrm{i}+12$, IRYCIS \\
\hline Preparación animal & $\begin{array}{l}\text { Contiguos al área } \\
\text { quirúrgica }\end{array}$ & $\begin{array}{l}\text { IRYCIS, IISBiodonostia, } \\
\text { IISBiocruces }\end{array}$ & En otras zonas comunes & $\mathrm{i}+12$, IISA \\
\hline $\begin{array}{l}\text { Área lavado y } \\
\text { esterilización material } \\
\text { quirúrgico }\end{array}$ & $\begin{array}{l}\text { Compartido con otras áreas } \\
\text { experimentación animal }\end{array}$ & $\begin{array}{l}\text { i+12, IRYCIS, } \\
\text { IISBiodonostia }\end{array}$ & $\begin{array}{l}\text { Exclusivo quirófano animal } \\
\text { grande }\end{array}$ & IISBiocruces, IISA \\
\hline Configuración pasillos & Pasillo Doble & $\begin{array}{l}\text { i+12, IISBiodonostia, } \\
\text { IISBiocruces }\end{array}$ & Pasillo Simple & IRYCIS, IISA \\
\hline
\end{tabular}

de las áreas quirúrgicas, se reflejan cuáles son las características comunes a la mayoría de los institutos y se identifica que institutos cumplen dichas características; además, se relacionan las características que son particulares o específicas y también se identifica que institutos las cumplen.

En el ámbito hospitalario, el bloque quirúrgico es una de las áreas críticas en cuanto a las exigencias de la calidad de aire, por lo que en general, se recomienda que esté ubicado en la última planta del edificio para garantizar la facilidad en el mantenimiento de la instalación. En cambio, en el caso de los quirófanos experimentales observamos cómo la mayoría de las áreas quirúrgicas experimentales se distribuyen en plantas con acceso directo desde el exterior, bien en plantas bajas o plantas semisótanos, ya que en general, lo que se prioriza en estos casos es la cercanía al área de estabulación de animal grande. El suministro de los animales grandes al centro es constante, y estos animales, que son bastante ruidosos y pueden ocasionar problemas de olores, hay que trasladarlos hasta la zona de estabulación. Es por ello por lo que conviene, en la medida de lo posible, y con el fin de evitar molestias al resto de los usuarios del instituto, que la zona de estabulación de animales grandes se sitúe en una planta con acceso directo al exterior y lo más cercano a este acceso.

En general, el área quirúrgica no se ha conseguido configurar como una unidad independiente del resto del centro de experimentación. Encontramos solo dos casos en los que la organización se realiza generando un área independizada (IIS Biocruces, IISA). En el resto de los casos, lo habitual es que el área quirúrgica se distribuya dentro del conjunto del centro de experimentación animal, de manera que las instalaciones generales tales como los vestuarios de cambio, los pasillos de distribución, almacenes varios y la sala de lavado y esterilización, sean comunes tanto para el área quirúrgica como para el centro de experimentación animal en general. Además, observamos cómo en algunos casos incluso se comparten locales como el destinado a la preparación animal y la preparación médica, por lo que se han tenido que implantar procedimientos de trabajo específicos para el uso de las salas con el fin de garantizar la asepsia necesaria en la unidad quirúrgica.

En estas áreas quirúrgicas se experimenta con animales grandes, siendo la especie animal más utilizada hoy en día el cerdo o cerdo en miniatura. Uno de los objetivos fundamentales de este tipo de quirófanos es la formación, de manera que están diseñados para que los profesionales médicos puedan adquirir la destreza necesaria para su aplicación en el ámbito hospitalario. Además, deben permitir abordar ensayos científicos de mayor complejidad como la valoración de dispositivos médicos, quirúrgicos y biomateriales, así como la realización de ensayos que requieran el trasplante de órganos.

El uso de estas áreas es una actividad totalmente programada en la que la circulación de los animales está protocolizada y siendo lo habitual que los animales sean terminales, o bien que interese ver la evolución durante un determinado periodo sin tener que estabularlos en el propio instituto durante un periodo largo de tiempo. Teniendo en cuenta esta premisa, vemos que existe una diferencia importante a tener en cuenta entre los quirófanos de experimentación animal y los quirófanos en los que se prestan servicios de salud, ya que, si bien 
en los quirófanos de hospitales es fundamental garantizar la protección del paciente contra los agentes nocivos, en el caso de la experimentación animal este aspecto no es tan crítico.

En cuanto a la configuración de los quirófanos, se observa que están diseñados como si fueran quirófanos hospitalarios con todos los locales de servicio necesarios para el correcto desarrollo de la actividad, si bien la fundamental característica diferencial es el número de mesas y el número de personas que nos encontramos en la sala de operaciones. Aunque existen centros en los que hay quirófanos con una única mesa quirúrgica, en la mayoría de los casos existen quirófanos con dos mesas (i+12, IRYCIS, IISBiodonostia, IISBiocruces, IISA). En los casos en los que el dimensionamiento de la sala lo ha permitido, con el paso del tiempo incluso se ha complementado la instalación con alguna mesa quirúrgica más (i+12).

Atendiendo a las características del diseño constructivo observamos como la tendencia de las áreas quirúrgicas experimentales es diseñarlos con los materiales de acabado similares a los que nos encontramos en el ámbito hospitalario (14). Siguiendo las directrices generales de facilidad de asepsia, los acabados que nos encontramos son continuos y lisos, con el mínimo número de uniones y fáciles de mantener y reparar, resistentes a la humedad y a los agentes de limpieza y desinfección, con encuentros curvos entre el pavimento y paramento vertical con el fin de garantizar una correcta limpieza y desinfección. Por otra parte, el equipamiento que habitualmente nos encontramos corresponde a lámparas cenitales, torre de cirugía y anestesia, paneles técnicos, equipos de video para retransmisión quirúrgica entre otros.

En todos los casos se dispone de un área de preparación médica contigua a los quirófanos, que en los casos en los que se ha podido, se ha utilizado para compartirlo como punto de acceso a varios quirófanos (IISBiocruces, IISA, IRICYS). Lo que no se ha conseguido en todos ellos es que esta zona de prequirófano sea de uso exclusivo para el personal médico, ya que vemos algunos casos en los que esta zona de preparación médica sirve de punto de acceso también de los animales (i+12, IRYCIS). Además, en un caso concreto observamos que se comparte también el uso como punto de acceso de los animales pequeños al área de microcirugía $(i+12)$ o como zona de paso obligado para acceso a los quirófanos tanto para el personal, como para los animales, el material estéril y la retirada de residuos (IRICYS).

La zona de preparación animal habitualmente se ubica en un local colindante al quirófano con acceso directo a este $(i+12$, IISBiodonostia, IISBiocruces). En otros casos, en vez de en locales que componen el bloque quirúrgico se ubica en locales del área de estabulación animal (IISA). Como casuísticas especiales se pueden mencionar el IIS Biodonostia, ya que, en este caso, el área de preparación animal es tanto para el animal pequeño como para el grande. Es por ello que para garantizar el correcto funcionamiento del centro se ha establecido un procedimiento de trabajo específico para el uso de esa sala y por lo tanto ambas actividades deben estar coordinadas para poder conseguir la asepsia necesaria.

Aunque lo habitual es que el almacén de material estéril sea un local compartido con la zona de preparación médica, existen algunos casos en los que este acceso se ha independizado de manera que existe; por una parte, una zona de preparación médica de acceso para el personal; y, por la otra un transfer de materiales para el acceso de material estéril al quirófano (i+12, IRICYS). Los locales de servicios relacionados con la actividad quirúrgica como son los locales de almacenamiento de residuos y cadáveres son áreas que habitualmente se comparten con los animales pequeños, y que se distribuyen en zonas periféricas al área quirúrgica.

En relación con la ubicación de los vestuarios, no en todos los casos se ha podido diseñar un núcleo para uso exclusivo del área quirúrgica, si bien observamos como el grado máximo de asepsia se consigue cuando el vestuario de cambio de personal está comunicado directamente con la zona de preparación médica o con un pasillo limpio de uso exclusivo del área quirúrgica (IISA, i+12, IISBiocruces).

Otra de las áreas necesarias para el desarrollo de la actividad es el área de lavado y esterilización del material quirúrgico. Aunque esta actividad mayoritariamente se desarrolla en locales exteriores al área quirúrgica, siendo habitual su uso compartido con otras plataformas del instituto, existen algunos casos en los que esta unidad se distribuye de forma exclusiva dentro del área quirúrgica (IISBiocruces, IISA).

Atendiendo a la configuración en función del tipo de pasillo seleccionado, vemos cómo no existe una tendencia clara que defina un modelo organizativo de pasillo simple o de pasillo doble. Teniendo en cuenta por una parte la organización y distribución de las áreas quirúrgicas comentadas anteriormente; y, por la otra, cómo se ha organizado la circulación del personal investigador, de los animales, del material estéril y de los residuos, en base al estudio realizado podemos establecer tres tipologías diferentes de organización de áreas quirúrgicas experimentales:

- Tipología 1: distribución con pasillo único. En este caso se centran los casos en los que para acceder a la zona de prequirófano y preparación animal el pasillo que se utiliza es el mismo, y, además, también se utiliza ese mismo pasillo para el suministro de material estéril y retirada de los residuos generados en el quirófano (IRYCIS; Figura 5).

- Tipología 2: distribución con pasillo doble. En este caso existen dos pasillos de circulación diferenciados; uno para el acceso del personal investigador y suministro de material limpio al quirófano; y, otro diferente desde el que se introduce a los animales y se retiran los residuos. (IISBiodonostia, IISA, IISBiocruces; Figuras 6, 7, 8).

- Tipología 3: distribución con pasillo doble. En este caso también existen dos pasillos de distribución, pero las circulaciones se ordenan de diferente manera al anterior; existe un pasillo para el acceso del personal investigador, material estéril y animales al quirófano, y otro diferente para la retirada de residuos del mismo (i+12; Figura 4).

En el caso de varios institutos (i+12 y IISA), observamos cómo a pesar de que este tipo de distribución la hemos considerado de doble pasillo por el hecho de que el acceso y la salida del quirófano de alguno de los elementos se hace por pasillos diferentes, lo cierto es que al final ambos pasillos acaban confluyendo en un mismo pasillo.

\section{CONCLUSIONES}

El uso de estas áreas destinada a la realización de prácticas quirúrgicas, es una actividad totalmente programada en la que la circulación de los animales está protocolizada, 
si bien existe una diferencia importante a tener en cuenta entre los quirófanos de experimentación animal y los quirófanos en los que se prestan servicios de salud, ya que, si bien en los quirófanos de hospitales es fundamental garantizar la protección del paciente contra los agentes nocivos, en el caso de la experimentación animal este aspecto no es tan crítico, ya que lo habitual es que los animales sean terminales, o bien que interese ver la evolución durante un determinado periodo sin tener que estabularlos en el propio instituto durante un periodo largo de tiempo, por lo que las exigencias de calidad de aire pueden ser más permisivas.

Observamos cómo el criterio utilizado para la implantación del área quirúrgica en el conjunto edificatorio es diferente al establecido en el ámbito hospitalario, siendo lo habitual que los quirófanos se distribuyan en plantas con acceso directo desde el exterior, ya que lo que se prioriza en estos casos es la cercanía al área de estabulación de animal grande.

Las instalaciones para experimentación con animal grande y pequeño tienen requerimientos de base muy diferentes debido fundamentalmente al tipo de investigación que se realiza, a las condiciones de asepsia y de bioseguridad exigibles, incluso a las exigencias de los propios animales de experimentación. Es por ello por lo que, aunque lo ideal sería poder diseñar un área de cirugía experimental totalmente independiente al área de experimentación de animal pequeño, la realidad es que por diversas circunstancias es muy común que ambas áreas compartan vestuarios, pasillos de distribución, almacenes varios y salas de lavado y esterilización. En algunos casos, incluso se comparten locales como el destinado a la preparación animal y a la preparación médica, lo que ha implicado implantar procedimientos de trabajo específicos que garanticen el correcto uso de la unidad quirúrgica.

Atendiendo a las características del diseño constructivo de los quirófanos, observamos cómo la tendencia de las áreas quirúrgicas experimentales es diseñarlos como si fueran quirófanos asistenciales con los locales de servicio necesarios, así como con los materiales de acabado similares a los que nos encontramos en el ámbito hospitalario, siguiendo las directrices generales de facilidad de asepsia y con el equipamiento correspondiente: lámparas cenitales, torre de cirugía y anestesia, paneles técnicos y equipos de video para retransmisión quirúrgica entre otros.

Los quirófanos se consideran salas de ambiente controlado por lo que es recomendable que en su diseño se consideren conceptos que garanticen el control requerido en estas salas. Ubicar la sala de preparación médica, la sala de preparación animal, así como el almacén de material estéril, incluso la salida de residuos colindante a los quirófanos, realiza la función de esclusa, lo que permite tener un control de las diferencias de presión entre salas, asegurando que el flujo de aire circule en la dirección adecuada, y, por lo tanto, evita la contaminación de agentes patógenos en la sala de operaciones. Así mismo, el grado de asepsia en la instalación mejora con la implantación de los vestuarios colindantes y con acceso directo al área quirúrgica o a la zona de preparación médica.

En cuanto al número de quirófanos que componen el área quirúrgica, lo habitual es que sea igual o superior a dos.
Estos quirófanos experimentales que son utilizados para la formación quirúrgica de los médicos, y con el fin de optimizar la superficie disponible en el centro, habitualmente son diseñados para instalar dos mesas de operaciones. En los casos en los que el dimensionamiento de la sala lo ha permitido, con el paso del tiempo incluso se ha complementado la instalación con alguna mesa quirúrgica más.

La configuración del área de cirugía experimental se puede organizar de diferentes maneras en función del criterio de distribución de pasillos seleccionado, de manera que podemos establecer tres tipologías diferentes de organización. Un tipo de distribución con pasillo único en el que tanto el personal investigador, el suministro de material limpio, los animales incluso los residuos acceden al quirófano por el mismo pasillo; un segundo tipo a base de distribución con pasillo doble en el que se separará el acceso del personal investigador y suministro del material limpio al quirófano; del de animales y los residuos; y, un tercer tipo a base de distribución con pasillo doble en el que el personal investigador, el suministro de material estéril y los animales son segregados de los residuos.

La implantación de un tipo u otro de diseño afecta directamente a la organización del personal y la disciplina de trabajo, por lo que en su decisión está habitualmente implicado tanto el equipo de diseño como los responsables del área de experimentación. Si bien la tipología de distribución con pasillo único es la solución más adecuada desde el punto de vista del aprovechamiento de la edificabilidad y la optimización de recursos, esta opción en la que se produce el cruce de los investigadores con los animales y del material limpio y sucio, requiere por parte de los profesionales el cumplimiento estricto de los procedimientos de trabajo establecidos, en aras a garantizar las condiciones asépticas necesarias en el quirófano. Es por ello, que la tendencia es realizar una distribución de manera que exista una segregación de circulaciones a la entrada del quirófano que permite independizar la circulación limpia de la sucia, considerando la limpia la destinada al personal investigador y suministro de material estéril, y la sucia la destinada a la circulación animal y retirada de residuos.

La mezcla de factores tales como el número de quirófanos, el número de mesas quirúrgicas en cada sala de operaciones, la ubicación de los locales de servicio, así como el tipo de configuración de pasillo seleccionado hacen que la organización de esta área no siga un patrón específico, resultando una distribución particular en cada caso.

Estas soluciones de organización y distribución tan variadas son el resultado obtenido de la combinación; por una parte, de las líneas de investigación establecidas por los responsables del área de experimentación animal; por otra parte, de las limitaciones impuestas por el conjunto edificatorio para la implantación del área quirúrgica; y, por último, de unos criterios de organización diferentes propuestos por los propios diseñadores.

La explosión tecnológica de los últimos años en el desarrollo de equipamiento médico cada vez más eficiente obliga a los profesionales médicos a adquirir la destreza necesaria para su aplicación posterior en el ámbito hospitalario en aras a mejorar la calidad de vida de la población. Lo que este estudio 
aporta es una visión de las diferentes soluciones adoptadas en el diseño de las áreas quirúrgicas experimentales en las que los profesionales médicos desarrollarán las habilidades. $\mathrm{Su}$ estudio permitirá enfocar los proyectos futuros con una visión amplia una vez conocidas las fortalezas y debilidades de cada solución adoptada, teniendo presente el objetivo final: intentar planificar, diseñar y construir lo mejor posible en aras a facilitar su funcionamiento eficiente, económico y seguro.

\section{AGRADECIMIENTOS}

Los autores quieren dejar constancia de su agradecimiento a todas las personas que con su intermediación nos han propiciado el acceso a cada uno de los institutos de investigación, así como a los responsables del área de experimentación animal de los institutos visitados, ya que, sin su confianza y dedicación, el presente trabajo no se podría haber llevado a término.

\section{REFERENCIAS}

(1) Parlamento europeo y del consejo. (2010). Directiva 2010/63/UE relativa a la protección de los animales utilizados para fines científicos. Diario oficial de la Unión Europea. L 276/33.

(2) National Research Council. (2011). Guide for the care and use of laboratory animals, p. 133, Washington, D.C.: National Academy Press.

(3) Real Decreto 279/2016, de 24 de junio, sobre acreditación de institutos de investigación biomédica o sanitaria. Boletín Oficial del Estado, núm. 161, de 5 de julio de 2016, pp. 47272 a 47283 . Recuperado de https://www.boe.es/boe/ dias/2016/07/05/pdfs/BOE-A-2016-6474.pdf

(4) Instituto de Salud Carlos III. Institutos de investigación sanitaria acreditados. https://www.isciii.es/QueHacemos/Financiacion/IIS/Paginas/Institutos-Acreditados-2015-2012.aspx

(5) Lainetti, E.B. F., Passos, L.A.C., Soares, C. R. J. y Nascimento, N. (2016). Conceptual proposal of a facility design for accomodating minipigs for pharmaceutical and radiopharmaceutical research, Journal of Civil Engineering and Architecture 10: 881-887, doi: 10.17265/1934-7359/2016.08.003

(6) Real Decreto 53/2013, de 1 de febrero, por el que se establecen las normas básicas aplicables para la protección de los animales utilizados en experimentación y otros fines científicos, incluyendo la docencia, núm. 34, de 8 de febrero de 2013, páginas 11370 a 11421. Recuperado de https://www.boe.es/boe/dias/2013/02/o8/pdfs/BOE-A-2013-1337.pdf

(7) Swindle, M.M., Makin, A., Herron, A.J., Clubb, F.J. Jr., Frazier, K.S. (2012). Swine as Models in Biomedical Research and Toxicology Testing. Veterinary Pathology 49 (2): 344-356, doi: 10.1177/0300985811402846

(8) Ministerio de Sanidad y política Social. (2009). Bloque Quirúrgico Estandares y Recomendaciones, p. 107, Madrid, Ministerio de Sanidad y Política Social Centro de Publicaciones.

(9) Martínez, M.L. (2014). Guía de buenas prácticas de circulación en el bloque quirúrgico, Madrid: Instituto Nacional de Gestión Sanitaria

(10) National Institutes of Health. (2016): Design requirements manual for biomedical laboratories and animal research facilities Rev1.4, p. 142, Bethesda (MD): NIH DRM. Recuperado de https://www.orf.od.nih.gov/PoliciesAndGuidelines/ Pages/DesignRequirementsManual2016.aspx

(11) Howard, H y Foucher, Y. K. F. (2009). Animal - Use Space. En Hessler J.R., Lehner N.D.M., Planing and Designing research animal facilities (pp. 204-214). London: Academic press, doi: https://doi.org/10.1016/B978-0-12-3695178.00019-0

(12) Canadian Council on Animal Care. (2003). Guidelines on: laboratory animal facilities - characteristics, design and development, Ottawa: CCAC Canadian Council on animal care.

(13) NHS States. (1991): Health Building Note 26 Operating Departmens, p. 8-9. London: HMSO.

(14) Prado, S., Lafebre, A. y Delgado, N. (2013). Guía para acabados interiores de hospitales, Quito: Ministerio de Salud Pública. 